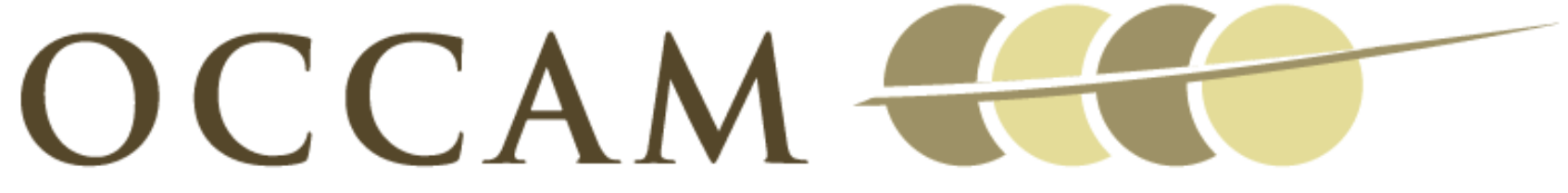

OXFORD CENTRE FOR COLLABORATIVE APPLIED MATHEMATICS

Report Number 10/39

Local synaptic signaling enhances the stochastic transport of motor-driven cargo in neurons

by

Jay Newby and Paul C. Bressloff

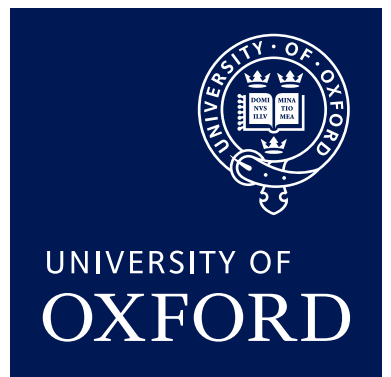

Oxford Centre for Collaborative Applied Mathematics Mathematical Institute 24 - 29 St Giles'

Oxford

OX1 3LB

England 



\title{
Local synaptic signaling enhances the stochastic transport of motor-driven cargo in neurons
}

\author{
Jay Newby and Paul C. Bressloff \\ Department of Mathematics, University of Utah, Salt Lake City, Utah 84112, \\ USA \\ Mathematical Institute, University of Oxford, 24-29 St. Giles', Oxford OX1 \\ $3 \mathrm{LB}, \mathrm{UK}$ \\ E-mail: bressloff@math.utah.edu
}

\begin{abstract}
The tug-of-war model of motor-driven cargo transport is formulated as an intermittent trapping process. An immobile trap, representing the cellular machinery that sequesters a motor-driven cargo for eventual use, is located somewhere within a microtubule track. A particle representing a motor-driven cargo that moves randomly with a forward bias is introduced at the beginning of the track. The particle switches randomly between a fast moving phase and a slow moving phase. When in the slow moving phase, the particle can be captured by the trap. To account for the possibility the particle avoids the trap, an absorbing boundary is placed at the end of the track. Two local signaling mechanisms - intended to improve the chances of capturing the target-are considered by allowing the trap to affect the tug-of-war parameters within a small region around itself. The first is based on a localized adenosine triphosphate (ATP) concentration gradient surrounding a synapse, and the second is based on a concentration of tau - a microtubule-associated protein involved in Alzheimer's disease - coating the microtubule near the synapse. It is shown that both mechanisms can lead to dramatic improvements in the capture probability, with a minimal increase in the mean capture time. The analysis also shows that tau can cause a cargo to undergo random oscillations, which could explain some experimental observations.
\end{abstract}

\section{Introduction}

A neuron relies heavily on microtubule transport to develop its asymmetric, extended morphology, and to maintain the functioning of its many cellular compartments. A neuron is usually composed of three main parts: a cell body (or soma) that contains the nucleus, a tubular protrusion called the axon that sends electrical signals to other neurons, and one or more highly branched tubular protrusions called dendrites that receive signals from other neurons. Many neurological disorders are characterized by a breakdown of the protein transport machinery. In particular, Alzheimer's disease - a lethal, degenerative neurological disease - is known to involve microtubule transport [1].

The motivating example of neuronal cargo transport we consider in this paper is mRNA transport in dendrites, which has been shown to be a key component of consolidation of long term synaptic plasticity [2]. However, the model presented here also applies to trasport of other types of cargo, such as mitochondrial trasport in axons and dendrites [3-6]. While many details regarding mRNA transport have been 
uncovered, how the motor-driven mRNA are delivered to specific synapses is still unclear. Synaptic spines in the dendrite are discrete structural units that compete with each other for resources transported from the soma. mRNA must be captured and temporarily sequestered from access by nearby competing synapses $[7,8]$. Once sequestered, the mRNA can either be consumed by a synapse undergoing plastic changes, or it can escape from sequestration and reenter the available pool of motordriven mRNA. Thus, delivery can be thought of as a two step process: first the mRNA is temporarily sequestered in an immobile pool and then it is later recruited by a synapse for translation. For the purposes of this paper, we will focus on the former. That is, we will consider "delivery" as capture and sequestration of a motor-driven cargo.

Microtubules - long filament tracks with a distinct $(+)$ and $(-)$ end-are useful for targeting resources to specific cellular compartments. In general, a given molecular motor will move with a bias toward a specific end of the microtubule; for example, kinesin moves toward the $(+)$ end and dynein moves toward the $(-)$ end. Microtubules are arranged throughout an axon or dendrite with a distribution of polarities: in axons and distal dendrites they are aligned with the (-) ends pointing to the soma (plus-endout) and in proximal dendrites they have mixed polarity $\ddagger[10,11]$. It is also known that many types of motor-driven cargo move bidirectionally along microtubules [7,8,12-14]. Together, these observations imply that cargo is transported by multiple kinesin and dynein motors. In proximal dendrites, it is also possible that one or more identical motors move a cargo bidirectionally by switching between microtubules with different polarities. In either case, it is well established that multiple molecular motors often work together as a motor-complex to pull a single cargo [15-17].

An open question remains as to how the many molecular motors pulling a cargo are coordinated [15]. One possibility is that the motors compete against each other in a tug-of-war where an individual motor interacts with other motors through the force it exerts on the cargo. If the cargo places a force on a motor in the opposite direction it prefers to move, it will be more likely to unbind from the microtubule. A recent biophysical model has shown that a tug-of-war can explain the coordinated behavior observed in experiment [18]. While this model provides an explanation for how a motor-complex works, it still remains to explain how such a motor-complex correctly delivers its cargo.

Recently, we extended the tug-of-war model to account for the position of the motor-complex on a microtubule track along with a target synapse somewhere within the track $[19,20]$. We included the possibility the motor-complex can find a hidden synaptic target if it is close enough to the target and in a slow moving state. This allowed us to interpret the random movement of the cargo as a particle undergoing an intermittent random search, where the particle transitions between a fast moving phase and a slow search phase. We used the model to calculate the delivery probability and the average delivery time to find the target and to explore how various biophysical parameters in the model could be tuned to optimize delivery.

In this paper we address a different question: how can the synapse change cellular conditions in a localized region around itself to improve cargo localization? Instead of an intermittent search where a randomly moving searcher must find an immobile hidden target, we can interpret this new scenario as a intermittent trapping process

¥ Certain invertebrates have been shown to have a minus-end-out distribution of dendritic microtubules [9] 
where an immobile trap must capture a randomly moving target. With this new formulation, we will use our model to calculate the capture probability and the mean first passage time (MFPT), defined as the average time necessary to capture the target, so that we can explore two possible signaling mechanisms a synapse might use to capture a nearby moving motor-driven cargo.

Adenosine triphosphate (ATP) - the energy source or fuel used by molecular motors to move along a microtubule - is a crucial component in cellular function and its availability is highly regulated. In a typical neuron, demands on ATP can be quite large especially near synapses where considerable levels of actin polymerization take place [21]. Since ATP concentration ([ATP]) is heavily buffered, a small region of intense ATP phosphorylation could create a sharp, localized [ATP] gradient, which would have a significant impact on a nearby motor-complex. Our analysis will show how an $[\mathrm{ATP}]$ concentration gradient surrounding the synaptic trap impacts the capture probability and MFPT.

Local [ATP] gradients affect the energy the motor-complex needs in order to pull its cargo. Another way to affect the motor-complex is to modify the interaction of the motor binding domains with the microtubule. For example, microtubule-associated proteins (MAPs) can bind to microtubules and effectively modify the free energy landscape of the motor-microtubule interactions [22,23]. Specifically, tau is a MAP found in the axon and is known to be a key player in Alzheimer's disease [24], and a recent study has found a link between tau phosphorylation and dopamine D1 receptor activation [25], but the function of tau in regulating mitochondrial transport is still unclear. Another important MAP, called MAP2, is similar in structure and function to tau, but is present in dendrites [26]. Moreover, MAP2 has been shown to be involved in activity-dependent changes in dendritic structure [27] and has also been shown to affect dendritic cargo transport [28]. Experiments have shown that tau and MAP2 can significantly affect the binding rate of kinesin to the microtubule [29-32]. This observation motivates the possibility that the presence of MAPs on the microtubule near a synapse can cause a motor-complex to disengage from the microtubule, allowing the cargo to be captured.

The structure of the paper is as follows. The tug-of-war model and how its various parameters depend on $[\mathrm{ATP}]$ and tau is presented in Section 2. We then formulate the tug-of-war model as an intermittent trapping process by including space so that we can consider capture by a hidden trap (Section 3). Finally, in Sections 4 and 5 we derive analytical approximations for the capture probability and MFPT. In Section 6, we use the analytical approximations combined with statistics extracted from Monte-Carlo simulations to explore how varying the ATP (Section 6.1) and tau/MAP2 (Section 6.2) concentration at a synapse affects the capture probability and MFPT.

\section{Tug-of-war model}

We begin by reviewing the tug-of-war model as it was originally formulated $[18,33]$. Suppose that there are two sets of motors bound to a given cargo. Each set of motors moves preferentially toward opposite ends of the microtubule. The case that we will consider in this paper is a set of $N_{+}$kinesin motors that prefer to move toward the microtubule $(+)$ end, and a set of $N_{-}$dynein motors that prefer to move toward the microtubule (-) end (see Fig. 1). When bound to a microtubule, each motor has a 
Local synaptic signaling enhances the stochastic transport of motor-driven cargo in neurons4

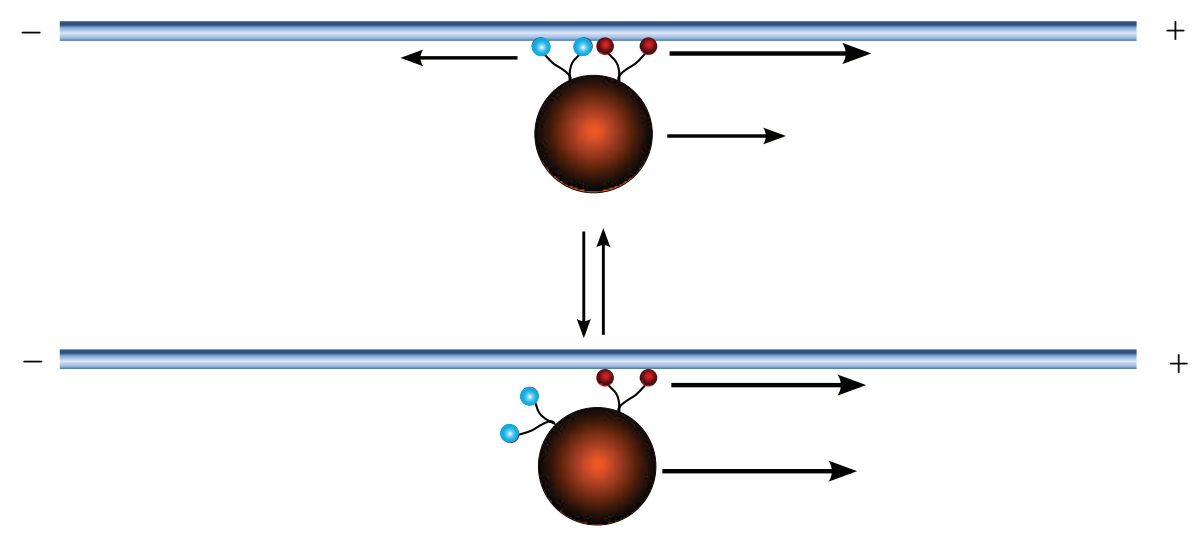

Figure 1. Diagram of the tug-of-war between different motors pulling a single cargo. In this example there is only a single motor in each population.

load-dependent velocity

$$
v(F)= \begin{cases}v_{f}\left(1-F / F_{s}\right) & \text { for } F \leq F_{s} \\ v_{b}\left(1-F / F_{s}\right) & \text { for } F \geq F_{s}\end{cases}
$$

where $F$ is the applied force, $F_{s}$ is the stall force satisfying $v\left(F_{s}\right)=0, v_{f}$ is the forward motor velocity in the absence of an applied force in the preferred direction of the particular motor, and $v_{b}$ is the backward motor velocity when the applied force exceeds the stall force. The unbinding rate is approximately an exponential function of the applied force

$$
\beta(F)=\beta_{0} \mathrm{e}^{\frac{F}{F_{d}}},
$$

where $F_{d}$ is the experimentally measured force scale on which unbinding occurs. On the other hand, the binding rate is taken to be independent of load:

$$
\pi(F)=\pi_{0} \text {. }
$$

Let $F_{c}$ denote the net load on the set of anterograde motors, which is taken to be positive when pointing in the retrograde direction. It follows that a single anterograde motor feels the force $F_{c} / n_{+}$, whereas a single retrograde motor feels the opposing force $-F_{c} / n_{-}$. Equations (2.2) and (2.3) imply that the binding and unbinding rates for the anterograde and retrograde motors are

$$
\begin{aligned}
& \beta_{ \pm}\left(n_{+}, n_{-}\right)=n_{ \pm} \beta_{0 \pm} \exp \left(F_{c} / n_{ \pm} F_{d \pm}\right) \\
& \pi_{ \pm}\left(n_{ \pm}\right)=\left(N_{ \pm}-n_{ \pm}\right) \pi_{0 \pm} .
\end{aligned}
$$

The cargo force $F_{c}$ is determined by the condition that all the motors move with the same cargo velocity $v_{c}$. Depending on the number of motors bound at a given time, either the anterograde or retrograde set of motors will be stronger. In the former case, the anterograde motors are dominant when $n_{+} F_{s+}>n_{-} F_{s-}$, and the net motion is in the anterograde direction, which is taken to be positive. Then, equation (2.1) implies that

$$
v_{c}=v_{f+}\left(1-F_{c} /\left(n_{+} F_{s+}\right)\right)=-v_{b-}\left(1-F_{c} /\left(n_{-} F_{s-}\right)\right) .
$$

We thus obtain a unique solution for the load $F_{c}$ and cargo velocity $v_{c}$ :

$$
F_{c}\left(n_{+}, n_{-}\right)=\left(\mathcal{F} n_{+} F_{s+}+(1-\mathcal{F}) n_{-} F_{s-}\right),
$$


where

$$
\mathcal{F}=\frac{n_{-} F_{s-} v_{f+}}{n_{-} F_{s-} v_{f+}+n_{+} F_{s+} v_{b-}}
$$

and

$$
v_{c}\left(n_{+}, n_{-}\right)=\frac{n_{+} F_{s+}-n_{-} F_{s-}}{n_{+} F_{s+} / v_{f+}+n_{-} F_{s-} / v_{b-}} .
$$

The corresponding formulas for the case where the backward motors are stronger, so that $n_{+} F_{s+}<n_{-} F_{s-}$, are found by interchanging $v_{f}$ and $v_{b}$.

Several groups have developed models of the [ATP] and force dependent motor parameters that closely match experiments for both kinesin [34,35] and dynein [36,37]. Based on these studies§ we take the forward velocity of a single motor under zero to have the Michaelis-Menten form

$$
v_{f \pm}([\mathrm{ATP}])=\frac{v_{f \pm}^{\max }[\mathrm{ATP}]}{[\mathrm{ATP}]+K_{m \pm}^{0}} .
$$

The backward velocity is small $\left(v_{b \pm}= \pm 0.006 \mu \mathrm{m} / \mathrm{s}\right)$ so that we can ignore its [ATP] dependence. The unbinding rate of a single motor under zero load can be determined using the $[\mathrm{ATP}]$ dependent average run length $L_{\rho \pm}([\mathrm{ATP}]) \|$. The mean time to detach from the microtubule is $v_{f \pm}([\mathrm{ATP}]) / L_{\rho \pm}([\mathrm{ATP}])$ so that

$$
\beta_{0 \pm}([\mathrm{ATP}])=\frac{v_{f \pm}^{\max }\left([\mathrm{ATP}]+K_{p \pm}\right)}{L_{\rho \pm}^{\max }\left([\mathrm{ATP}]+K_{m \pm}^{0}\right)}
$$

The binding rates are determined by the time necessary for an unbound motor to diffuse within range of the microtubule and bind to it, which is assumed to be independent of both load and $[\mathrm{ATP}]$. Finally, the $[\mathrm{ATP}]$ dependent stall force is given by

$$
F_{s \pm}([\mathrm{ATP}])=F_{s \pm}^{0}+\frac{\left(F_{s \pm}^{\max }-F_{s \pm}^{0}\right)[\mathrm{ATP}]}{K_{s \pm}+[\mathrm{ATP}]} .
$$

Experiments have shown that the presence of tau or MAP2 on the microtubule can significantly alter the dynamics kinesin; specifically, by reducing the rate at which kinesin binds to the microtubule [32]. Moreover, the tau- and MAP2-dependent kinesin binding rate have the same form [30]. It has also been shown that, at the low tau concentrations affecting kinesin, dynein is relatively unaffected by tau [31]. We are unaware of any experiments that have looked at how MAP2 affects dynein, but given the similarities between tau and MAP2, it is likely that dynein will be similarly resistant to low concentrations of MAP2. Since more experimental evidence exists to quantify the effect of tau on kinesin binding, we use the tau-dependent kinesin binding rate instead of that of MAP2 - even though we are modeling cargo transport in dendrites.

As we will show, the binding rate of kinesin has the greatest affect on the dynamics of the motor-complex, so that as a first approximation, we can ignore how any other parameters might vary in the presence of tau. Based on these experiments, we model

$\S$ Others later refined the kinesin model to include a more detailed description of the chemomechanical stepping process [38-40]. However, we proceed with the minimal kinesin model as the results obtained here do not change significantly when incorporating these more complex models.

$\|$ We are making the assumption that $\left\langle v_{f} / L\right\rangle=\left\langle v_{f}\right\rangle /\langle L\rangle$, which is justified on spatial scales that we consider. For more details see [40]. 
the affect of local concentrations of tau bound to the microtubule by introducing the tau concentration dependent binding rate for kinesin given by:

$$
\pi_{0}(\tau)=\frac{\pi_{0}^{\max }}{1+e^{-\gamma\left(\tau_{0}-\tau\right)}},
$$

where $\tau$ is the dimensionless ratio of tau per microtubule dimer and $\pi_{0}^{\max }=5 \mathrm{~s}^{-1}$. The remaining parameters are found by fitting the above function to experimental data [32], so that $\tau_{0}=0.19$ and $\gamma=100$.

Table 1. Tug-of-war parameter values

\begin{tabular}{cll}
\hline \multicolumn{1}{c}{ Parameter } & Kinesin & Dynein \\
\hline Max. antero. velocity $v_{f}^{\max }(\mu \mathrm{m} / \mathrm{s})$ & $1[34]$ & $0.7[36]$ \\
Retro. velocity $v_{b}(\mu \mathrm{m} / \mathrm{s})$ & $0.006[41]$ & 0.006 \\
ATP equil. const. $K_{m}^{0}(\mu M)$ & $79[34]$ & $38[36,37]$ \\
Max run length $L^{\max }(\mu \mathrm{m})$ & $0.86[35]$ & $1.5[36]$ \\
ATP equil. const. $K_{p}(\mu M)$ & $3.13[35]$ & $1.5[36,37]$ \\
Low ATP stall force $F_{s}^{0}(p N)$ & $5.5[34]$ & $0.22[36,37]$ \\
High ATP stall force $F_{s}^{\max }(p N)$ & $8[34]$ & $1.24[36,37]$ \\
ATP equil. const. $K_{s}(p N)$ & $100[34]$ & $480[36,37]$ \\
Unbinding force scale $F_{d}(p N)$ & $3[34]$ & $3[36]$ \\
Low tau binding rate $\pi_{0}\left(\mathrm{~s}^{-1}\right)$ & $1.6[32]$ & - \\
Max tau binding rate $\pi_{0}^{\max }\left(\mathrm{s}^{-1}\right)$ & $5[32]$ & - \\
Tau dep. binding rate scale $\gamma$ & $100[32]$ & - \\
Tau scale $\tau_{0}$ & $0.19[32]$ & - \\
\hline
\end{tabular}

A list of parameter values consistent with experiments are found in Table 1.

\section{Generalized intermittent trapping model}

The tug-of-war model [18] considers the stochastic dynamics associated with transitions between different internal states $\left(n_{+}, n_{-}\right)$of the motor-cargo complex, without specifying the spatial position of the cargo along a one-dimensional track. This defines a Markov process with a corresponding master equation for the time evolution of the probability distribution $p\left(n_{+}, n_{-}, t\right)$. In order to apply the model to the intermittent trapping problem, it is necessary to construct a differential ChapmanKolmogorov (CK) equation for the probability density $p\left(n_{+}, n_{-}, x, t\right)$ that the cargo is in the internal state $\left(n_{+}, n_{-}\right)$and has position $x$ at time $t$.

The intermittent trapping problem for the tug-of-war model is formulated as follows. Suppose a target particle is moving along a one dimensional track of length $L$ having started at some location $0 \leq x_{0} \leq L$. The track represents a segment of dendrite, which can be a single branch or a segment of a long branch. To model an entire dendrite one must consider a tree-network, which has been developed in a previous paper [42]. In general, the track can consist of one or more linked microtubules, but we will consider a single effective microtubule and, for simplicity, ignore details regarding jumping between parallel microtubules. At the left boundary of the track we impose a reflecting boundary, which can represent the soma or a branch point; however, we also assume the particle's motion is biased in the anterograde 
direction, which means the reflecting boundary will have little effect. At the right boundary, we impose an absorbing boundary, which represents degradation of the cargo or sequestration by competing synapses. That is, the combination of biased transport, low levels of degradation, and sequestration by competing synapses, the probability of returning to the trap after traveling a sufficient distance beyond it will be approximately zero. Indeed, the possibility of failure of the trap to capture the particle is a key feature of our analysis, and the absorbing boundary is included to explore this.

A trap is introduced somewhere within the track at location $x=X$ so that if the target particle is within a distance $l$ of the trap and in a receptive state it can be captured by the trap at a rate $k_{0}$. For the tug-of-war model, we assume that the target particle is in a slowly-moving receptive state, where it is able to be captured if the number of anterograde and retrograde motors are equal $\left(n_{+}=n_{-}\right)$; whereas, it is in a fast moving non-receptive state when $n_{+} \neq n_{-}$. Since kinesin and dynein have different biophysical properties, the velocity $v_{c}(n, n)$ in states with equal numbers of opposing motors engaged will be small but not identically zero.

In order to write down the CK equation, it is convenient to introduce the label $i\left(n_{+}, n_{-}\right)=\left(N_{+}+1\right) n_{-}+\left(n_{+}+1\right)$ and set $p\left(n_{+}, n_{-}, x, t\right)=p_{i\left(n_{+}, n_{-}\right)}(x, t)$. We then have an $n$-component probability density vector $\boldsymbol{p} \in \mathbb{R}^{n}$ with $n=\left(N_{+}+1\right)\left(N_{-}+1\right)$. The corresponding differential CK equation takes the form [43]

$$
\partial_{t} \boldsymbol{p}=A(x) \boldsymbol{p}-\mathcal{L}(\boldsymbol{p}),
$$

where the space-dependent matrix $A(x) \in \mathbb{R}^{n \times n}$ contains the transition rates between each of the $n$ internal motor states. The differential operator $\mathcal{L}$ has the structure

$$
\mathcal{L}=\left[\begin{array}{ccccc}
\mathcal{L}_{1} & 0 & \ldots & & 0 \\
0 & \mathcal{L}_{2} & 0 & \ldots & 0 \\
\vdots & & \ddots & & \vdots \\
& & & \mathcal{L}_{n-1} & 0 \\
0 & \cdots & & 0 & \mathcal{L}_{n}
\end{array}\right]
$$

where the scalar operators are given by

$$
\mathcal{L}_{i}=\left[\kappa_{i}(x)+\partial_{x} v_{i}(x)\right] .
$$

Here, $v_{i}(x)$ is the velocity of internal state $i=i\left(n_{+}, n_{-}\right)$and $\kappa_{i}(x)$ is the rate of target capture at location $x$. Assuming that the trap is at location $X$ and can affect the environment within a region of half-width $l$ around itself, we set $\kappa_{i}(x)=k_{i} \chi(x)$ and

$$
v_{i}(x)=v_{c}\left(n_{+}, n_{-} ; S(x)\right), \quad i=i\left(n_{+}, n_{-}\right),
$$

where

$$
S(x)=S_{1}+S_{2} \chi(x)
$$

is the signal (either $\tau$ or $[\mathrm{ATP}]$ ) concentration, and

$$
\chi(x)=\left\{\begin{array}{lc}
1, & \text { if }|x-X|<l \\
0, & \text { otherwise }
\end{array}\right.
$$

The constants $S_{1,2}$ are the concentrations away from the trap and near the trap, respectively. The target particle can only captured if it is within range and in a slowly-moving receptive state so that the capture rates are taken to be

$$
k_{i}=\left\{\begin{array}{cc}
k_{0} & \text { if } n_{+}=n_{-} \\
0 & \text { otherwise }
\end{array}\right.
$$


Although the velocities in any state where at least one of each motor type is bound will be slow, extending the set of nonzero capture rates does not alter the qualitative conclusions and does not significantly affect the quantitative results obtained in this paper. The components $a_{i, j}(S(x)), i, j=1, \ldots, n$, of the state transition matrix $A(x)$ are given by the corresponding binding/unbinding rates of equations (2.4) and (2.5). The space dependence is given implicitly through the spatially-varying signal concentration $S(x)(3.5)$. Setting $i=i\left(n_{+}, n_{-}\right)$, the non-zero off-diagonal terms are (suppressing the $S(x)$ dependence)

$$
\begin{aligned}
& a_{i, j}=\pi_{+}\left(n_{+}-1\right) \text { for } j=i\left(n_{+}-1, n_{-}\right) \\
& a_{i, j}=\pi_{-}\left(n_{-}-1\right), \text { for } j=i\left(n_{+}, n_{-}-1\right) \\
& a_{i, j}=\beta_{+}\left(n_{+}+1, F_{c}\right), \text { for } j=i\left(n_{+}+1, n_{-}\right),
\end{aligned}
$$

and

$$
a_{i, j}=\beta_{-}\left(n_{-}+1, F_{c}\right), \text { for } j=i\left(n_{+}, n_{-}+1\right) .
$$

The diagonal terms are then given by $a_{i, i}=-\sum_{j \neq i} a_{j, i}$. We note that at any fixed location $x$, the Markov process defined by the CK equation

$$
\partial_{t} \varrho=A(x) \varrho,
$$

admits a stationary distribution $\hat{\boldsymbol{p}}(x)$ such that $A(x) \hat{\boldsymbol{p}}(x)=0$, and $\sum_{i=1}^{n} \hat{p}_{i}(x)=1$.

Finally, it is necessary to specify the initial conditions and boundary conditions at the ends $x=0, L$. We will impose an initial condition of the form $\boldsymbol{p}(x, 0)=\delta(x) \hat{\boldsymbol{p}}$. At the origin we impose the reflecting boundary condition

$$
\sum_{i=1}^{n} v_{i}(0) p_{i}(0, t)=0, \quad \forall i=1, \cdots, n
$$

and the auxiliary condition

$$
\frac{p_{1}(0, t)}{\hat{p}_{1}(0)}=\frac{p_{i}(0, t)}{\hat{p}_{i}(0)}
$$

for all $i=2, \cdots, n$ such that $v_{i}(0)>0$. At the other end of the interval $(x=L)$ we impose the absorbing boundary condition

$$
p_{i}(L, t)=0,
$$

for all $i=1, \cdots, n$ such that $v_{i}(L)<0$.

\section{Model reduction}

To analyze the model we compute the capture probability and the MFPT. The capture probability is given by

$$
\mathcal{P}=k_{0} \int_{0}^{\infty} \mathcal{J}(t) d t
$$

and the MFPT is given by

$$
\mathcal{T}=\frac{k_{0}}{\mathcal{P}} \int_{0}^{\infty} t \mathcal{J}(t) d t,
$$

where the probability flux into the trap is given by

$$
\mathcal{J}(t)=\int_{X-l}^{X+l} d x \sum_{n_{+}, n_{-}} p\left(n_{+}, n_{-}, x, t\right) \delta_{n_{+}, n_{-}} .
$$


For ease of notation we have suppressed the dependence on initial conditions.

Although it would be possible in principal to calculate $\mathcal{P}$ and $\mathcal{T}$ analytically for the full model by following along similar lines to previous studies of 3 -state models [44,45], the analysis is considerably more involved due to the complexity of the molecular motor model. Therefore, we follow a different approach here by carrying out a quasi-steadystate (QSS) reduction of the differential CK equation (3.1) based on a QSS reduction previously applied to the spatially-homogeneous process $[19,20]$. The reduced model is described by a scalar Fokker-Planck (FP) equation with an extra inhomogeneous decay term accounting for target detection. Within this approximation, the capture probability and MFPT can be expressed in terms of the Laplace transformed solution of the FP equation. Others have considered similar reductions for jump-velocity processes, with space-dependent velocity states and state transitions $[46,47]$. In these models, the jump-velocity process describes a cell moving in response to a spatiallyvarying chemotactic substance. The most significant difference in the model considered here is that the signal $S(x)$ is spatially localized.

Suppose that we fix the units of space and time by setting $l=1$ and $l / v(1,0)=1$, which corresponds to non-dimensionalizing the CK equation (3.1) by performing the rescaling $x \rightarrow x / l$ and $t \rightarrow t v(1,0) / l$. Furthermore, we assume that for the given choice of units $a_{i j}=\mathcal{O}(1 / \varepsilon)$ and $\mathcal{L}_{i j}=\mathcal{O}(1)$, for some small parameter $\varepsilon \ll 1$, and set $A=\varepsilon^{-1} \widehat{A}$. We can then carry out a QSS reduction of the dimensionless CK equation

$$
\partial_{t} \boldsymbol{p}=\frac{1}{\varepsilon} \widehat{A}(x) \boldsymbol{p}-\mathcal{L}(\boldsymbol{p}) .
$$

The QSS reduction is carried out using a standard projection method $\boldsymbol{9}$. If the state velocities $v_{i}$ are small and the transition rates $a_{i j}$ are fast, then the CK equation will rapidly evolve toward the stationary distribution $\hat{\boldsymbol{p}}$ of the space-clamped Markov process (3.12). The solution can then be written as

$$
\boldsymbol{p}(x, t)=u(x, t) \hat{\boldsymbol{p}}(x)+\varepsilon \boldsymbol{w}(x, t),
$$

where

$$
u(x, t)=\sum_{i=1}^{n} p_{i}(x, t), \quad \sum_{i=1}^{n} w_{i}(x, t)=0 .
$$

The spatially-varying signal concentration defined by (3.5) imposes a discontinuity in the state transition rates, which leads to a discontinuity in the stationary distribution $\hat{\boldsymbol{p}}$. This means that the discontinuity will also perturb the internal-state distribution of the solution away from the stationary distribution near the point of discontinuity. Thus, for the anzatz (4.5) to be valid, we must require that $S_{2}=\mathcal{O}(\varepsilon)$ so that the jump in $\hat{\boldsymbol{p}}$ is also $\mathcal{O}(\varepsilon)$.

For brevity we omit the details of the perturbation method here (for full details see $[19,20])$. First, it is convenient to define the mean of a vector $\mathbf{v}$ with respect to the stationary distribution $\hat{\boldsymbol{p}}$ according to

$$
\langle\mathrm{v}(x)\rangle \equiv \mathbf{v}^{T}(x) \hat{\boldsymbol{p}}(x) .
$$

We also define the vectors $\boldsymbol{v}(x)=\left(v_{1}(x), \cdots, v_{n}(x)\right)^{T}$ and $\boldsymbol{k}=\left(k_{1}, \cdots, k_{n}\right)^{T}$, which contain the state velocities and capture rates, respectively. After applying the QSS reduction we obtain the Fokker-Planck (FP) equation for $u$

$$
\partial_{t} u=-\lambda u-\partial_{x}(V u)+\partial_{x}\left(D \partial_{x} u\right)
$$

I For general details on projection methods in the context of stochastic model reduction see [43]. 
with the $x$-dependent parameters

$$
\begin{aligned}
& \lambda(x)=\chi(x)\left(\langle k\rangle-\varepsilon \boldsymbol{k}^{T} \boldsymbol{q}(x)+\mathcal{O}\left(\varepsilon^{2}\right)\right) \\
& V(x)=\langle v(x)\rangle+\varepsilon \chi(x)\left(-\boldsymbol{k}^{T} \boldsymbol{r}(x)-\boldsymbol{v}^{T}(x) \boldsymbol{q}(x)+\mathcal{O}(\varepsilon)\right) \\
& D(x)=\varepsilon \boldsymbol{v}^{T}(x) \boldsymbol{r}(x)+\mathcal{O}\left(\varepsilon^{2}\right) \chi(x) .
\end{aligned}
$$

We note that $\lambda, V$, and $D$ depend on $x$ and are piecewise constant on the domain $(0, L)$, with jump discontinuities at $x=X \pm l$. The vectors $\boldsymbol{r}(x)$, and $\boldsymbol{q}(x)$ satisfy the following equations for all $x \in[0, L]$

$\widehat{A}(x) \boldsymbol{r}(x)=-\left(\left(\langle v(x)\rangle-v_{1}(x)\right) \hat{p}_{1}(x), \cdots,\left(\langle v(x)\rangle-v_{n}(x)\right) \hat{p}_{n}(x)\right)^{T}$

$\widehat{A}(x) \boldsymbol{q}(x)=-\left(\left(\langle k\rangle-k_{1}\right) \hat{p}_{1}(x), \cdots,\left(\langle k\rangle-k_{n}\right) \hat{p}_{n}(x)\right)^{T}$

Although the matrix $\widehat{A}(x)$ will be singular, it can be shown that solutions to the above equations exist. We obtain a unique solution by imposing the condition

$$
\sum_{i=1}^{n} r_{i}(x) \equiv 0, \quad \sum_{i=1}^{n} q_{i}(x) \equiv 0 .
$$

At $\mathcal{O}(\varepsilon)$, the drift velocity $V$ picks up additional terms within the trapping region in order to compensate for the flux into the trap. In order to compute the $\mathcal{O}(\varepsilon)$ contributions to $\lambda, V$, and $D$, the rank deficient equations (4.12)-(4.13) can be solved numerically using the full singular value decomposition of the matrix $\widehat{A}$ (for details see $[19,20])$.

Initial conditions for the reduced FP equation (4.8) are chosen from the stationary distribution $\hat{\boldsymbol{p}}$ so that

$$
u(x, 0)=\delta(x) .
$$

Boundary conditions corresponding to (3.13) and (3.15) are

$$
D \partial_{x} u-\left.V u\right|_{x=0}=0, \quad u(L, t)=0 .
$$

For simplicity, the initial condition and the reflecting boundary condition can be combined to get the inhomogeneous Robin condition

$$
D \partial_{x} u-\left.V u\right|_{x=0}=-1 \text {. }
$$

\section{MFPT and capture probability}

To analyze the efficiency of the generalized intermittent trapping process, we will use the FP equation (4.8) to calculate the capture probability $P$ and MFPT $T$, which approximate the exact capture probability $\mathcal{P}$ and MFPT $\mathcal{T}$ defined by (4.1) and (4.2), respectively. Let $Q(t)=\int_{0}^{L} u(x, t) d x$ be the total probability that the particle is still located in the domain $0<x<L$ at time $t$. After integrating equation (4.8) with respect to $x$ and using the reflecting boundary conditions (4.16), we have

$$
\frac{\partial Q}{\partial t}=-\int_{X-l}^{X+l} \lambda(x) u(x, t) d x+D \partial_{x} u(L, t) .
$$

It follows that the total flux into the trap is

$$
J(t)=\int_{X-l}^{X+l} \lambda(x) u(x, t) d x,
$$


The capture probability, having started at $x=0$ at time $t=0$, is then

$$
P=\int_{0}^{\infty} J(t) d t
$$

and the corresponding MFPT is

$$
T=\frac{\int_{0}^{\infty} t J(t) d t}{\int_{0}^{\infty} J(t) d t} .
$$

Consider the Laplace transform of the probability flux $J$,

$$
\Upsilon(s) \equiv \widetilde{J}(s)=\int_{0}^{\infty} \mathrm{e}^{-s t} J(t) d t
$$

Taylor expanding the integral with respect to the Laplace variable $s$ shows that

$$
\begin{aligned}
\Upsilon(s) & =\int_{0}^{\infty} J(t)\left[1-s t+s^{2} t^{2} / 2-\ldots\right] d t \\
& =P\left[1-s T+\frac{s^{2}}{2} T^{(2)}-\ldots\right],
\end{aligned}
$$

assuming that the moments

$$
T^{(n)}=\frac{\int_{0}^{\infty} t^{n} J(t) d t}{\int_{0}^{\infty} J(t) d t}
$$

are finite. Thus, $\Upsilon(s)$ can be viewed as a generating function for the moments of the conditional first passage time distribution [48]. Equations (5.2) and (5.5) imply that

$$
\Upsilon(s)=\int_{X-l}^{X+l} \lambda(x) \widetilde{U}(x, s) d x,
$$

where $\widetilde{U}(x, s)$ is the Laplace transform of $u(x, t)$. Once the generating function has been computed the capture probability and MFPT are given by

$$
P=\Upsilon(0), \quad T=-\frac{\Upsilon^{\prime}(0)}{\Upsilon(0)} .
$$

Hence, we can proceed by solving the Laplace transformed FP equation (4.8) to determine $\widetilde{U}(x, s)$. Substituting the result into equation (5.8) then allows us to extract $P$ and $T$ using equation (5.9).

Now that we have a way of extracting the mean first-passage time and capture probability, we need only solve for the Laplace transform of the function $\widetilde{U}$. Because of the piecewise-constant parameters in the FP equation (4.8), we divide the interval $[0, L]$ into three regions and solve the equation separately in each region. Region one is the interval $(0, X-l)$, region two corresponds to the immobile trap on the interval $(X-l, X+l)$, and region three is the interval $(X+l, L)$. We subscript functions and parameters belonging to each region by $\rho=1,2,3$. For simplicity, each region will have its own local coordinate system $x_{\rho}$, where

$$
x_{1}=x, \quad x_{2}=x-(X-l), \quad x_{3}=x-(X+l) .
$$

Laplace transforming the FP equation (4.8) yields

$$
D_{\rho} \partial_{x}^{2} \widetilde{U}_{\rho}\left(x_{\rho}, s\right)-V_{\rho} \partial_{x} \widetilde{U}_{\rho}\left(x_{\rho}, s\right)-\left(s+\lambda_{\rho}\right) \widetilde{U}_{\rho}\left(x_{\rho}, s\right)=0,
$$

where the initial condition $u(x, 0)=\delta_{\rho, 1} \delta(x)$ is accounted for by imposing the Robin boundary condition

$$
V_{1} \widetilde{U}_{1}(0, s)-D_{1} \partial_{x} \widetilde{U}_{1}(0, s)=1 .
$$


Other boundary conditions are as follows. In region one we impose the open boundary condition

$$
\widetilde{U}_{1}\left(L_{1}, s\right)=\Phi_{a}(s)
$$

where $\Phi_{a}(s)$ is an unknown function. In region two we impose the open boundary conditions

$$
\widetilde{U}_{2}(0, s)=\Phi_{a}(s), \text { and } \quad \widetilde{U}_{2}\left(L_{2}, s\right)=\Phi_{b}(s),
$$

where $L_{2}=2 l$ and $\Phi_{b}(s)$ is an unknown function. Finally, in region three we impose the open boundary condition

$$
\widetilde{U}_{3}(0, s)=\Phi_{2}(s)
$$

at $x_{3}=0$ and the absorbing boundary condition

$$
\widetilde{U}_{3}\left(L_{3}, s\right)=0
$$

at $x_{3}=L_{3}=L-X-l$. We can then solve for the unknown functions $\Phi_{a, b}(s)$ by imposing conservation of flux at the boundary between region one and two and the boundary between region two and three. This results in a $2 \times 2$ system of equations for $\Phi_{a, b}(s)$.

For ease of notation, we will suppress the explicit functional dependence on the Laplace variable $s$. The eigenvalues are given by

$$
\mu_{\rho, \pm}=\frac{V_{\rho}}{2 D_{\rho}} \pm \eta_{\rho}, \quad \eta_{\rho}=\frac{\sqrt{V_{\rho}^{2}+4\left(s+\lambda_{\rho}\right) D_{\rho}}}{2 D_{\rho}}
$$

To solve (5.11), we use linearity to construct the solution from a superposition of sub-solutions satisfying individual boundary conditions. The solution satisfying a reflecting boundary condition at the origin is given by

$$
\phi\left(x_{1}\right)=\left(\mu_{1,-} D_{1}-V_{1}\right) \mathrm{e}^{\mu_{1,+} x_{1}}-\left(\mu_{1,+} D_{1}-V_{1}\right) \mathrm{e}^{\mu_{1,-} x_{1}},
$$

and the solution satisfying an absorbing boundary condition at the origin is given by

$$
\psi_{\rho}\left(x_{\rho}\right)=\mathrm{e}^{\mu_{\rho,+} x_{\rho}}-\mathrm{e}^{\mu_{\rho,-} x_{\rho}} .
$$

Using these sub-solutions we construct the full solution to (5.11) in each region by applying appropriate boundary conditions to get

$$
\begin{aligned}
& \widetilde{U}_{1}\left(x_{1}, s\right)=\widehat{F}_{1}\left(x_{1}\right)+\Phi_{a} F_{1}\left(x_{1}\right) \\
& \widetilde{U}_{2}\left(x_{2}, s\right)=\Phi_{a} \widehat{F}_{2}\left(x_{2}\right)+\Phi_{b} F_{2}\left(x_{2}\right) \\
& \widetilde{U}_{3}\left(x_{3}, s\right)=\Phi_{b} \widehat{F}_{3}\left(x_{3}\right)
\end{aligned}
$$

where

$$
\begin{aligned}
& \widehat{F}_{1}\left(x_{1}\right)=\frac{-\psi_{1}\left(x_{1}-L_{1}\right)}{D_{1} \psi_{1}^{\prime}\left(-L_{1}\right)-V_{1} \psi_{1}\left(-L_{1}\right)}, \quad F_{1}\left(x_{1}\right)=\frac{\phi\left(x_{1}\right)}{\phi\left(L_{1}\right)} \\
& \widehat{F}_{2}\left(x_{2}\right)=\frac{\psi_{2}\left(x_{2}-L_{2}\right)}{\psi_{2}\left(-L_{2}\right)}, \quad F_{2}\left(x_{2}\right)=\frac{\psi_{2}\left(x_{2}\right)}{\psi_{2}\left(L_{2}\right)} \\
& \widehat{F}_{3}\left(x_{3}\right)=\frac{\psi_{3}\left(x_{3}-L_{3}\right)}{\psi_{3}\left(-L_{3}\right)} .
\end{aligned}
$$

To finalize the solution we must determine the unknown functions $\Phi_{a, b}$. We define the flux operator according to

$$
\mathfrak{J}_{\rho}[f](x) \equiv D_{\rho} f^{\prime}(x)-V_{\rho} f(x) .
$$


Imposing conservation of flux yields a system of two equations for the two unknowns $\Phi_{a, b}$

$$
\begin{aligned}
& \mathfrak{J}_{1}\left[\widehat{F}_{1}\right]\left(L_{1}\right)+\Phi_{a} \mathfrak{J}_{1}\left[F_{1}\right]\left(L_{1}\right)=\Phi_{a} \mathfrak{J}_{2}\left[\widehat{F}_{2}\right](0)+\Phi_{b} \mathfrak{J}_{2}\left[F_{2}\right](0) \\
& \Phi_{a} \mathfrak{J}_{2}\left[\widehat{F}_{2}\right]\left(L_{2}\right)+\Phi_{b} \mathfrak{J}_{2}\left[F_{2}\right]\left(L_{2}\right)=\Phi_{b} \mathfrak{J}_{3}\left[\widehat{F}_{3}\right](0) .
\end{aligned}
$$

To simplify the solution, we introduce the following abbreviation for the various fluxes

$$
\begin{aligned}
& g_{\rho} \equiv \mathfrak{J}_{\rho}\left[\widehat{F}_{\rho}\right]\left(L_{\rho}\right), \quad h_{\rho} \equiv \mathfrak{J}_{\rho}\left[F_{\rho}\right]\left(L_{\rho}\right) \\
& \bar{g}_{\rho} \equiv \mathfrak{J}_{\rho}\left[F_{\rho}\right](0), \quad \bar{h}_{\rho} \equiv \mathfrak{J}_{\rho}\left[\widehat{F}_{\rho}\right](0),
\end{aligned}
$$

Solving (5.28) for $\Phi_{b}$ yields

$$
\Phi_{b}=\frac{-g_{2}}{H_{2}} \Phi_{a}
$$

where,

$$
H_{\rho}=h_{\rho}-\bar{h}_{\rho+1}
$$

Substituting (5.31) into (5.27) yields

$$
\Phi_{a}=\frac{-g_{1}}{H_{1}-\frac{g_{2} \bar{g}_{2}}{H_{2}}}
$$

Combining (5.33) and (5.31) yields the desired result

$$
\widetilde{U}_{2}\left(x_{2}, s\right)=\frac{-g_{1}}{H_{1}-\frac{g_{2} \bar{g}_{2}}{H_{2}}}\left(\widehat{F}_{2}\left(x_{2}\right)-\frac{g_{2}}{H_{2}} F_{2}\left(x_{2}\right)\right) .
$$

The capture probability and MFPT, given by the formula (5.9), can now be calculated by substituting the above solution into (5.8).

\section{Results and discussion}

In the last section, we formulated the tug-of-war model as an intermittent random trapping process where an immobile trap at location $X$ changes its environment in a small region of radius $l$ around itself to improve its chances of capturing a randomly moving target. The immobile trap represents the localized machinery used by a synapse to recruit newly synthesized mRNA. A cargo attached to a multiple motorcomplex consisting of $N_{-}$dynein motors and $N_{+}$kinesin motors is coordinated through a tug-of-war competition. The means by which the trap changes the environment around itself to improve target capture represents a signaling mechanism.

In this section, we will explore two signaling mechanisms based on a localized [ATP] gradient and a localized concentration of tau. To model these two signals, we use the $[\mathrm{ATP}]$ and $\tau$ dependent tug-of-war parameters (see Table 1 ) to carry out a QSS reduction for two sets of parameters (we assume that environment to the left and right of the trap region are identical so that in terms of the notation from Section 5 we have $V_{1}=V_{3}$ and $\left.D_{1}=D_{3}\right)$. The first set of QSS parameters $\left(\lambda_{1}, V_{1}\right.$, and $\left.D_{1}\right)$ are valid away from the immobile trap, and the second set $\left(\lambda_{2}, V_{2}\right.$, and $\left.D_{2}\right)$ are valid within a distance $l$ of the trap (since the particle can only be captured near the trap, we set $\lambda_{1,3}=0$ and $\lambda=\lambda_{2}$ ). We define the drift velocity and diffusivity as functions of $x$

$$
V(x)=V_{1}+\Delta V \chi(x), \quad D(x)=D_{1}+\Delta D \chi(x),
$$

where $\chi(x)$ is the indicator function defined in (3.6), $\Delta V=V_{2}-V_{1}$, and $\Delta D=D_{2}-D_{1}$. The capture probability $P$ and MFPT $T$ are then found by substituting these QSS parameters into the expression for $\widetilde{U}_{2}(5.34)$, and using the result in the formula for the generating function (5.8). 


\subsection{ATP signal}

Kinesin and dynein both require ATP as fuel to move their cargo along a microtubule. In a dendrite, local ATP concentration gradients may form as a result of actin polymerization at an active synapse. We would like to see how a reduction in available ATP near a synapse improves cargo localization. To analyze the model in terms of [ATP], we select parameters for dynein and kinesin according to Table 1, with the binding rate for kinesin set to $\pi_{0}=\pi_{0}^{\max }=5$. We perform the QSS reduction to obtain $V_{1}$ and $D_{1}$ for fixed [ATP] at $10^{3} \mu M$ away from the target. Then, [ATP] is reduced within a distance $l$ of the trap so that the second set of QSS parameters $\left(\lambda([\mathrm{ATP}]), V_{2}([\mathrm{ATP}]), D_{2}([\mathrm{ATP}])\right)$ are functions of the reduced [ATP] . The effect of lowering $[\mathrm{ATP}]$ near the trap is characterized by the $[\mathrm{ATP}]$ dependence of the capture rate $\lambda$, the drift velocity $V$, and the diffusivity $D$ (see Fig. 2). The capture rate $\lambda$ is
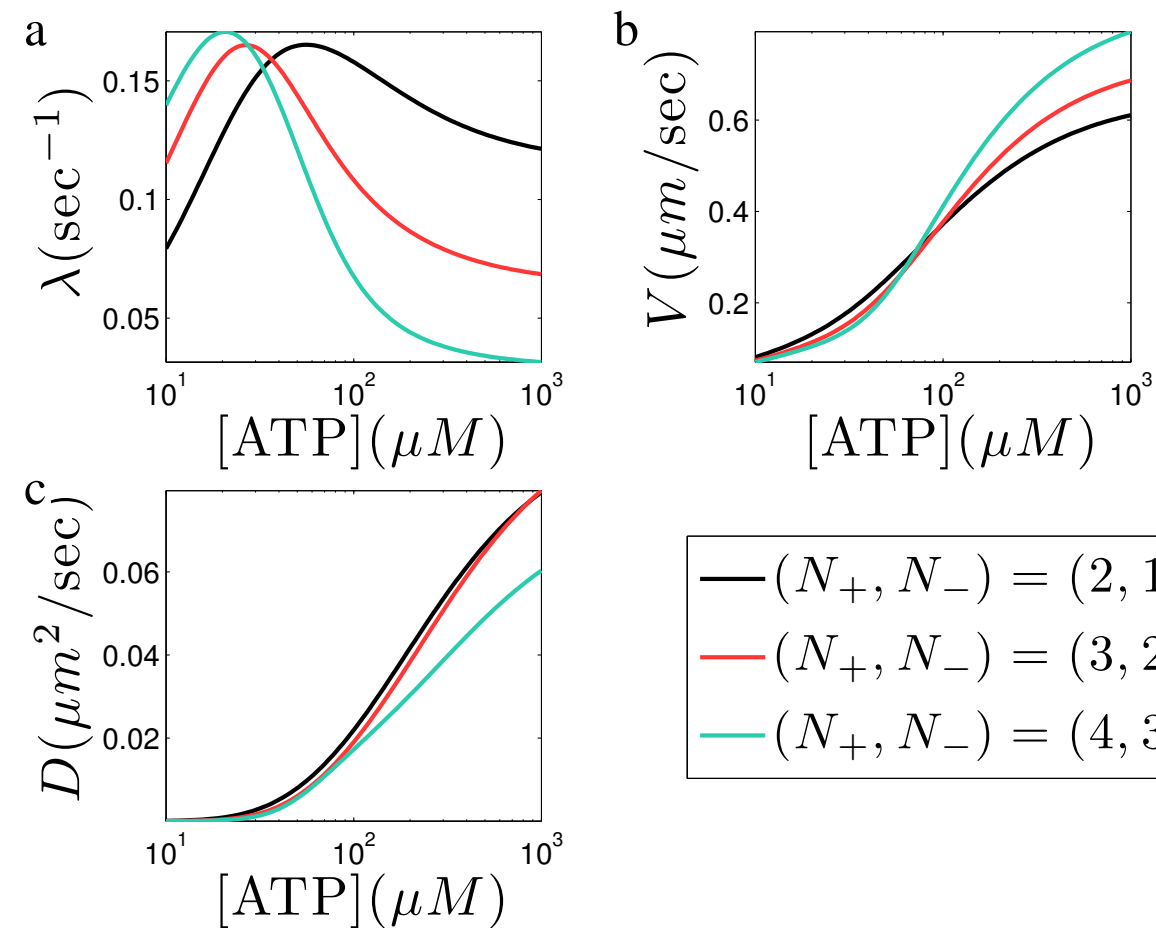

$$
\begin{array}{r}
-\left(N_{+}, N_{-}\right)=(2,1) \\
-\left(N_{+}, N_{-}\right)=(3,2) \\
-\left(N_{+}, N_{-}\right)=(4,3)
\end{array}
$$

Figure 2. QSS parameters as a function of [ATP $]$ obtained from the tug-of-war parameters in Table 1. a) The effective capture rate $\lambda$. b) The drift velocity $V$. c) The diffusivity $D$.

a decreasing function at high [ATP] because the motor-complex is more likely to be in a mobile, non-receptive state with more fuel around (Fig. 2a). A maximum in the capture rate at low $[\mathrm{ATP}]$ suggests that the motor-complex will be more receptive to capture if the trap were to lower [ATP]. However, reducing the available amount of fuel will also decrease the average speed of the motor-complex. Recall that kinesin is much stronger than dynein so that, even if the motor-complex is comprised of equal numbers of kinesin and dynein motors, the motion of the motor-complex will always have a directional bias. At low $[\mathrm{ATP}]$ kinesin looses much of its strength; therefore, 
the coordinated motion of the motor-complex becomes less biased, the drift velocity is reduced, and the individual motors move more slowly so that the diffusivity is reduced (Fig. 2b,c). Thus, slowing the particle down will increase the capture rate by making it spend more time near the trap, but does so at the cost of raising the MFPT.

The [ATP]-dependent peak in the capture rate and reduction of the drift velocity depend on the motor configuration $\left(N_{+}, N_{-}\right)$. As the number of kinesin and dynein motors is increased, the maximum drift velocity, at $[\mathrm{ATP}]=10^{3} \mu M$, increases. The drift velocity for the motor configuration $(4,3)$ drops at a faster rate as $[\mathrm{ATP}]$ is reduced, compared to the other two configurations. Increasing the number of motors also causes the peak capture rate to occur at a lower [ATP]. This behavior suggests the effect of changing [ATP] on the capture probability and MFPT will depend on the motor configuration. The capture probability $\mathcal{P}$ and MFPT $\mathcal{T}$ (black curves) resulting from changing the $[\mathrm{ATP}]$ near the target are shown in Fig. 3 . These results clearly

$\mathbf{a}$
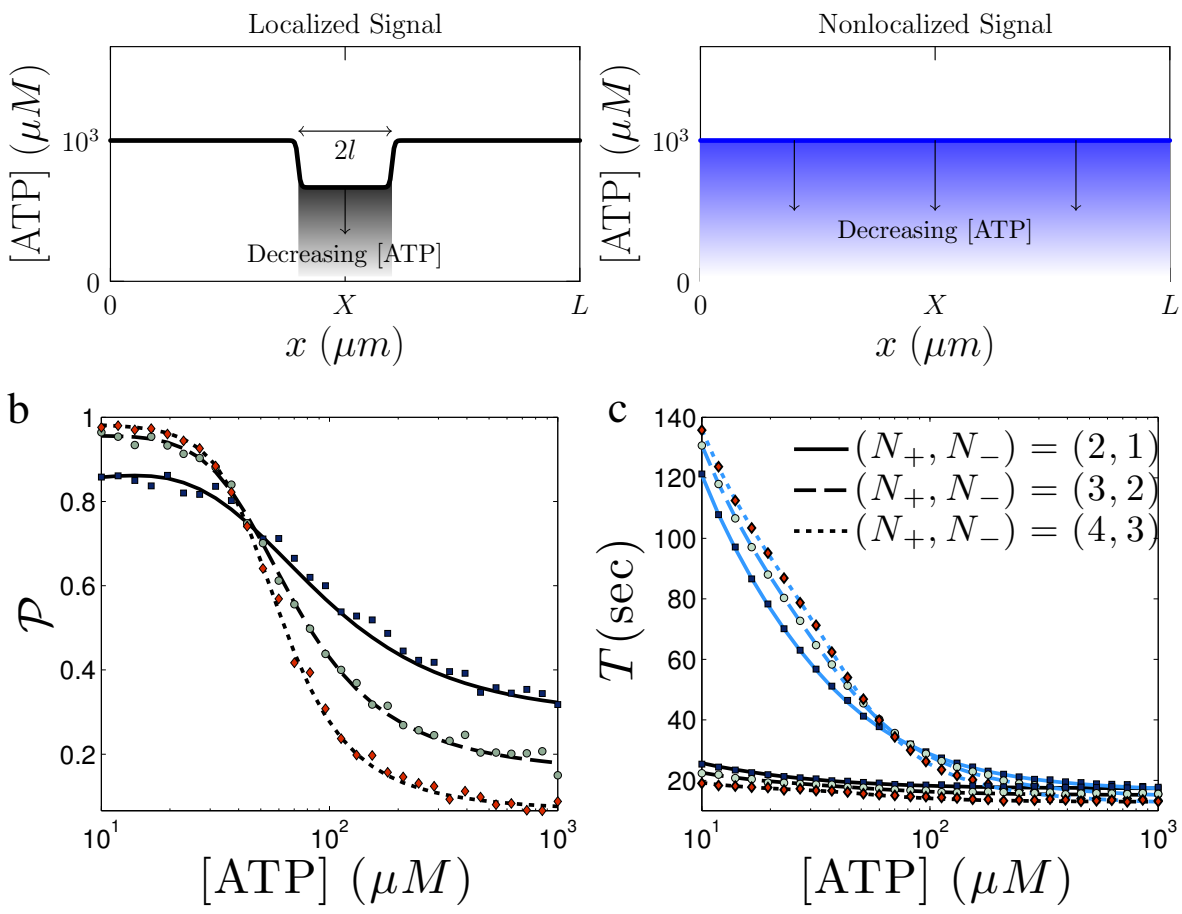

Figure 3. The capture probability $\mathcal{P}$ and MFPT $\mathcal{T}$ as functions of [ATP] using QSS and tug-of-war parameters from Fig. 2. a) The concentration of ATP is lowered near the trap for the localized signal (black solid line) and lowered everywhere for the nonlocalized signal (blue solid line) b) Analytical approximation $P$ (solid lines) and the results of Monte-Carlo simulations (symbols). c) Analytical approximation $T$ (solid lines) along with averaged MonteCarlo simulations (symbols). The black curves show the MFPT for the case where the signal is localized, so that [ATP] is held fixed at $10^{3} \mu M$ away from the synapse and decreased within a distance $l=2 \mu \mathrm{m}$ of the synapse. The blue curves show the case where the signal is not localized, so that ATP is decreased throughout the whole domain. The length of the microtubule track is $L=20 \mu \mathrm{m}$, and the synaptic trap is located at $X=10 \mu \mathrm{m}$. The capture rate is $k_{0}=0.5 \mathrm{~s}^{-1}$.

show that lowering the $[\mathrm{ATP}]$ near the trap can improve the capture probability. 
As expected, the level of response to the ATP signal depends on $\left(N_{+}, N_{-}\right)$. The idea is to balance the number of kinesin and dynein motors so that the drift velocity is high at background levels of $[\mathrm{ATP}]$ and low near the trap where $[\mathrm{ATP}]$ is reduced. This requires the signal to be localized to the trap so that the cargo velocity is high for most of the distance the cargo must travel to reach the trap and only reduced for a short time while the cargo is near the trap. Without the signal localization, a global reduction in $[\mathrm{ATP}]$ would result in a significant increase in the MFPT.

In contrast, the capture probability is approximately independent of how far outside the trapping radius the signal extends. For a 1D search, the cargo will encounter the trap with probability one. Because the motion of the particle is biased in the forward direction, the particle will be unlikely to re-encounter the trap after passing it up. Thus, only the behavior of the particle within the trapping radius will significantly affect the capture probability. This means that we can restrict the region where the signal is applied to minimize the MFPT, without affecting the capture probability. To see this, we compare the MFPT for a localized ATP reduction to a reduction of ATP throughout the whole domain (blue curves) in Fig. 3c. For each choice of motor configuration, the MFPT is increased by an order of magnitude in the absence of signal localization. The corresponding results for the capture probability in Fig. 3b are not shown, as the analytical approximations and $\mathrm{MC}$ simulations for the two cases are indistinguishable.

\subsection{Tau signal}

Tau and MAP2 can bind to a microtubule and alter the free energy landscape of the interactions between a molecular motor and the microtubule. Experiments have shown that tau and MAP2 can alter the binding rate of kinesin to the microtubule [29-32]. Because the effect of tau and MAP2 on the binding rate of kinesin is functionally identical [30], we will drop the distinction between the two and simply refer to them both as tau. Theoretical models have also explored how tau affects the binding rate of kinesin and how this effect alters the dynamics of transport by teams of motors [49-51], but have not explored stochastic cargo delivery.

To explore how tau might affect the capture probability and MFPT, we use the tau dependent tug-of-war parameters from Table 1 and assume that ATP concentration is held fixed at $10^{3} \mu M$. This yields QSS parameters $\lambda, V$, and $D$ that are functions of $\tau$ (see Fig. 4). In contrast with the [ATP] signal, the effective capture rate $\lambda$ changes very little with tau concentration. The capture rate and drift velocity also depend much less on the motor configuration - a fact that suggests the tau signal is more robust than the $[\mathrm{ATP}]$ signal. The most significant alteration in the behavior of the motor-complex is the change in the drift velocity $V$. The drift velocity switches sign (Fig. 4b) when $\tau$ is increased past a critical point. By reducing the binding rate of kinesin, the dynein motors become dominant, causing the motor-complex to move in the opposite direction.

To calculate the capture probability and MFPT, we first compute the QSS parameters $\left(V_{1}\right.$, and $\left.D_{1}\right)$ with $\tau$ fixed at zero. The second set of QSS parameters $\left(\lambda(\tau), V_{2}(\tau), D_{2}(\tau)\right)$ are valid within a distance $l$ of the trap. In Fig. 5, we plot the capture probability $\mathcal{P}$ and the MFPT $\mathcal{T}$ as a function of $\tau$ near the target. As $\tau$ is increased above the critical level $\tau_{0}=0.19$, we see a sharp increase in $\mathcal{P}$, confirming that $\tau$ can improve the capture probability. We also see a small rise in the MFPT $\mathcal{T}$, which is comparable to rise from the $[\mathrm{ATP}]$ signal (Fig. 3 ). Note we restrict the 
Local synaptic signaling enhances the stochastic transport of motor-driven cargo in neurons17

a
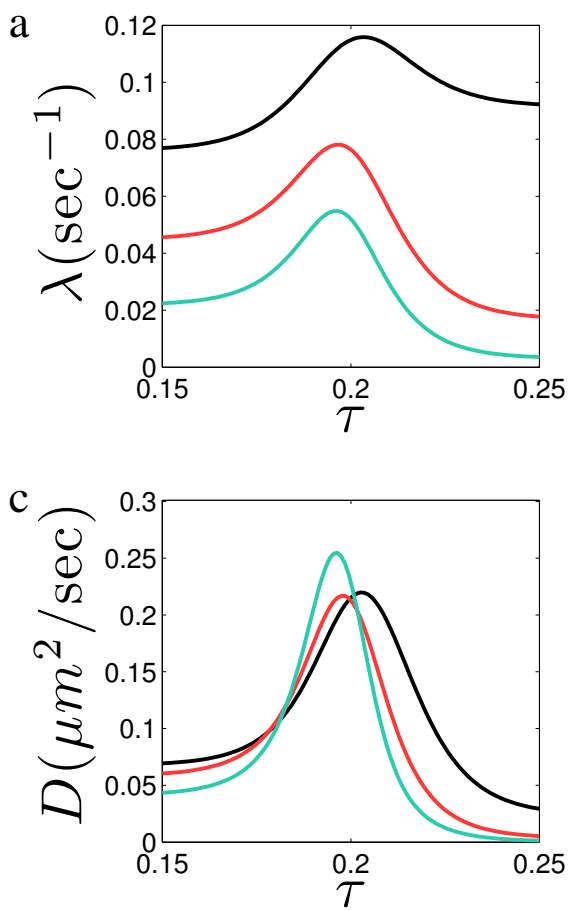

b

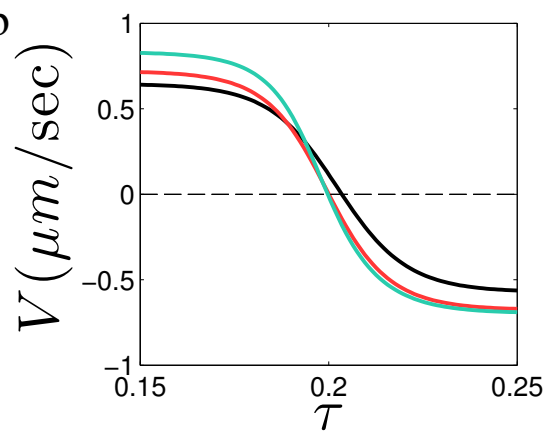

Figure 4. Parameters from the QSS reduction as a function of tau concentration obtained from the tug-of-war parameters in Table 1, with [ATP] fixed at $10^{3} \mu M$. a) The effective capture rate $\lambda$. b) The drift velocity $V$. c) The diffusivity $D$.
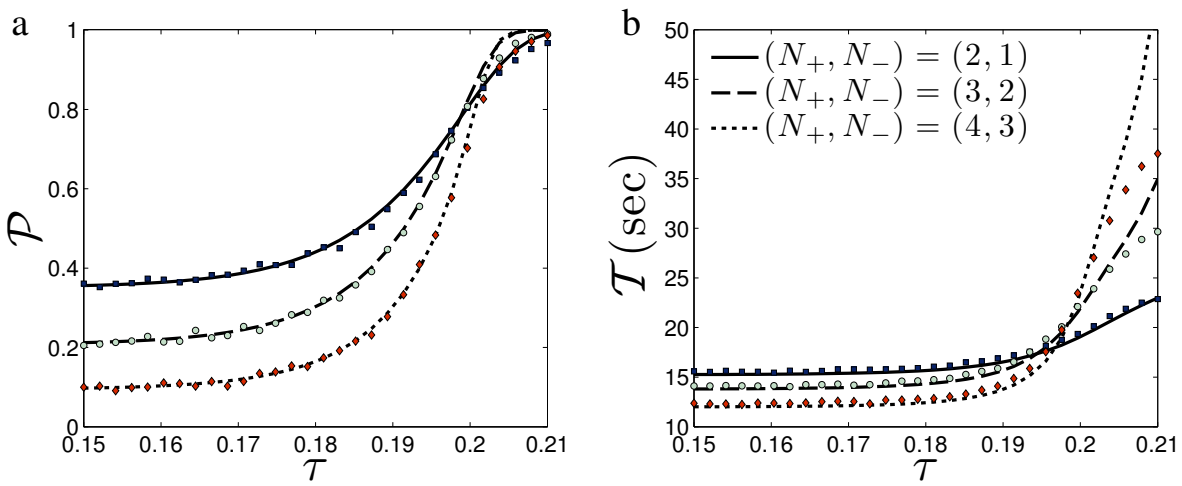

Figure 5. Effect of adding tau to the target on the capture probability $\mathcal{P}$ and MFPT $\mathcal{T}$ using parameters from Fig. 4. a) The analytical approximation $P$ (solid line) and results from Monte-Carlo simulation. b) The analytical approximation $T$ along with averaged Monte-Carlo simulations. The synaptic trap is located at $X=10 \mu m$, the trapping region has radius $l=2 \mu m$, and the microtubule track has length $L=20 \mu \mathrm{m}$. The capture rate is taken to be $k_{0}=0.5 \mathrm{~s}^{-1}$. 
results to a maximum value of $\tau=0.21$ because the capture probability is near unity at this level and the QSS approximation loses accuracy at higher tau levels - a fact we explore in more detail below.

Changing the sign of the drift velocity near the trap has many intriguing implications. Most notably, tau can create stochastic oscillations in the motion of the motor-complex. As a kinesin driven cargo encounters the tau-coated trapping region the motors unbind at their usual rate and can't rebind. Once the dynein motors are strong enough to pull the remaining kinesin motors off the microtubule, the motorcomplex quickly transitions to $(-)$ end directed transport. Then as the dynein driven cargo leaves the tau-coated region, kinesin motors are allowed to reestablish $(+)$ end directed transport until the motor-complex returns to the tau-coated region. This process repeats until the motor-complex is able to move forward past the tau-coated region. Interestingly, particle tracking experiments have observed oscillatory behavior during mRNA transport in dendrites [7,8]. In these experiments, motor-driven mRNA granules move rapidly until encountering a fixed location along the dendrite where they slightly overshoot then stop, move backwards, and begin to randomly oscillate back and forth. After a period of time - lasting about one minute - the motor-driven mRNA stops oscillating and resumes fast ballistic motion.

The initial overshoot can be explained by a rapidly moving motor-complex with several kinesin motors engaged encountering a patch of tau on the microtubule. The motor-complex would continue moving forward until enough of the kinesin motors unbind to allow the dynein motors to take over and move the cargo backwards. Tauinduced random oscillations can also explain why the motor-driven mRNA sometimes resumes fast ballistic transport. While oscillating, the motor-complex is moving within an effective potential well, which is reminiscent of the hysteresis effect from a time-varying external force predicted by Müller et. al. [52]. However, in our case, the random oscillations are not caused by an external force, but rather the signal's influence on the transition rates and motor velocities, which can be thought of as an effective force generating an effective potential well.

Consider a drift velocity $V$ that arises due to a constant force $F_{\text {ext }}=\vartheta V$ acting on a Brownian particle under dissipation. Without loss of generality assume that $\vartheta=1$, then the potential (see Fig. 6) arising from a piecewise-constant force is given by

$$
\begin{aligned}
\Psi(x) & =\int_{X-l}^{x} V\left(x^{\prime}\right) d x^{\prime} \\
& =\left\{\begin{array}{cc}
-V_{1}(x-X+l), & x<X-l \\
-V_{2}(x-X+l), & X-l<x<X+l \\
-V_{1}(x-X-l)-2 l V_{2}, & X+l<x
\end{array}\right.
\end{aligned}
$$

Depending on the length of the region influenced by the trap, and the magnitude of the drift velocities, the time spent in the potential well can be quite long. Suppose that a Brownian particle starts at the bottom of the potential well. The corresponding mean exit time (MET) is given by

$$
\begin{aligned}
M_{B} & =\int_{X-l}^{X+l} \exp \left(-\frac{\Psi(y)}{D(y)}\right) d y \int_{-\infty}^{y} \frac{\exp \left(\frac{\Psi(z)}{D(z)}\right)}{D(z)} d z \\
& =\int_{0}^{2 l} \mathrm{e}^{-\frac{V_{1}}{D_{1}} y} d y\left(\frac{1}{D_{1}} \int_{-\infty}^{0} \mathrm{e}^{\frac{V_{1}}{D_{1}} z} d z+\frac{1}{D_{2}} \int_{0}^{y} \mathrm{e}^{\frac{V_{2}}{D_{2}} z} d z\right)
\end{aligned}
$$



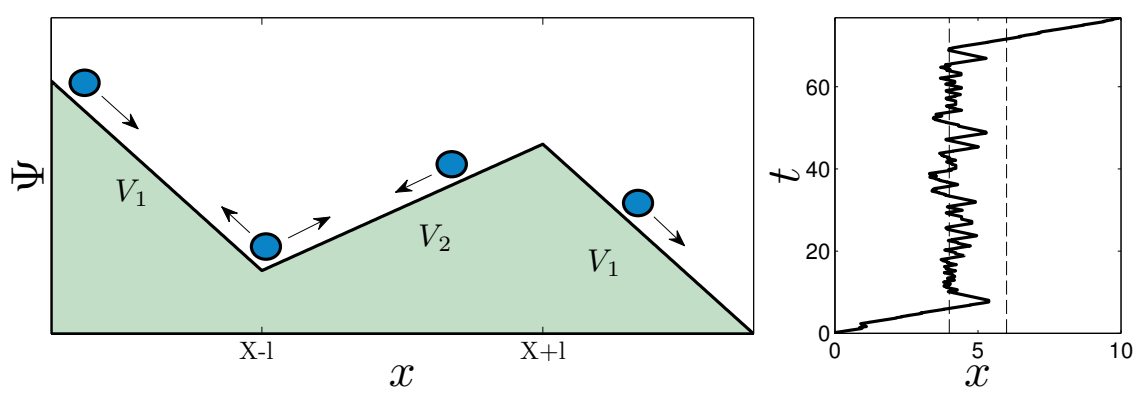

Figure 6. Diagram showing the effective potential well created by a region of tau coating a microtubule, and a representative trajectory showing random oscillations.

$$
=\frac{2 l D_{2}}{\nu}\left(\mathrm{e}^{\frac{\nu}{D_{2}}}-1\right)\left(\frac{1}{V_{1}}+\frac{2 l}{\nu}\right)-\frac{4 l^{2}}{\nu},
$$

where $\nu=-V_{2} 2 l$ is the depth of the well. In general, the MET will be an exponentially increasing function of the depth of the well. This means that if we wish to estimate the MET with the QSS reduction, any error generated by the approximation will also grow exponentially. Because the transition rates contained in the matrix $A$ contains a discontinuity at $X-l$, the stationary distribution $\hat{\boldsymbol{p}}$ is also discontinuous at this point. The QSS reduction assumes that the probability distribution instantly transitions from one stationary distribution to the next, when in fact it takes some time for this to occur. The higher order diffusion term in the FP equation (4.8) can approximate the behavior of the particle when its internal-state distribution remains within a small $\mathcal{O}(\varepsilon)$ neighborhood of the stationary solution. If the drift velocities all point in the same direction, the error produced by this assumption is small — as was the case for the ATP signal. However, since the drift velocity changes sign for the tau signal, the particle will spend a significant amount of time crossing back and forth over the turning point $x_{\text {turn }}$ where $V\left(x_{\text {turn }}\right)=0$ (see Fig. $6 \mathrm{~b}$ ). For this reason, we expect $M_{B}$, which is based on the QSS reduction, to be a poor approximation for the MET. Unfortunately, one cannot correct for this using higher order terms in the perturbation expansion since contributions from higher moments of the propagator become significant. Thus, the full model must be solved to accurately calculate the MET. Using techniques based on the backward CK equation, it can be shown that when starting at position $x_{0}$ in state $i$, the MET satisfies the following equation

$$
v_{i} \partial_{y} M_{i}(y)+\sum_{j=1}^{n} \hat{a}_{j, i}(y) M_{j}(y)=-1, \quad i=1, \cdots, n,
$$

where the state velocities $v_{i}$ are given by (3.4). The transition rates are defined by

$$
\hat{a}_{i, j}(y)=a_{i, j}^{-}+\left(a_{i, j}^{+}-a_{i, j}^{-}\right) \chi(y),
$$

where $a_{i, j}^{+}=a_{i, j}(\tau), a_{i, j}^{-}=a_{i, j}(0)$, and the $\tau$ dependent transition rates $a_{i, j}(\tau)$ are given by (3.8)-(3.11) along with $\tau$ dependent parameters in Table 1. For simplicity, we have defined the transition rates $\hat{a}_{i, j}(y)$ so that the minimum of the potential well is located at $y=0$. Boundary conditions for the above equation are as follows. First, the solution grows linearly as $y \rightarrow=-\infty$ so that for all $i=1, \cdots, n$

$$
M_{i}(y) \propto-x, \quad \text { as } y \rightarrow-\infty .
$$


Second, the solution is continuous at $y=0$ so that

$$
\lim _{h \rightarrow 0}\left[M_{i}(y)\right]_{y=-h}^{y=h}=0 .
$$

Finally, at the far boundary of the tau-coated region, the MET vanishes for internal states with a corresponding positive velocity, so that

$$
M_{i}(2 l)=0,
$$

for all $i=1, \cdots, n$ such that $v_{i}>0$. The averaged solution to (6.7), defined as $\langle M\rangle=\sum_{i=1}^{n} M_{i} \hat{p}_{i}$, is shown in Fig. 7 along with results from averaged Monte-Carlo simulations. Note that we have reduced the detection radius $l$ by half in order to reduce

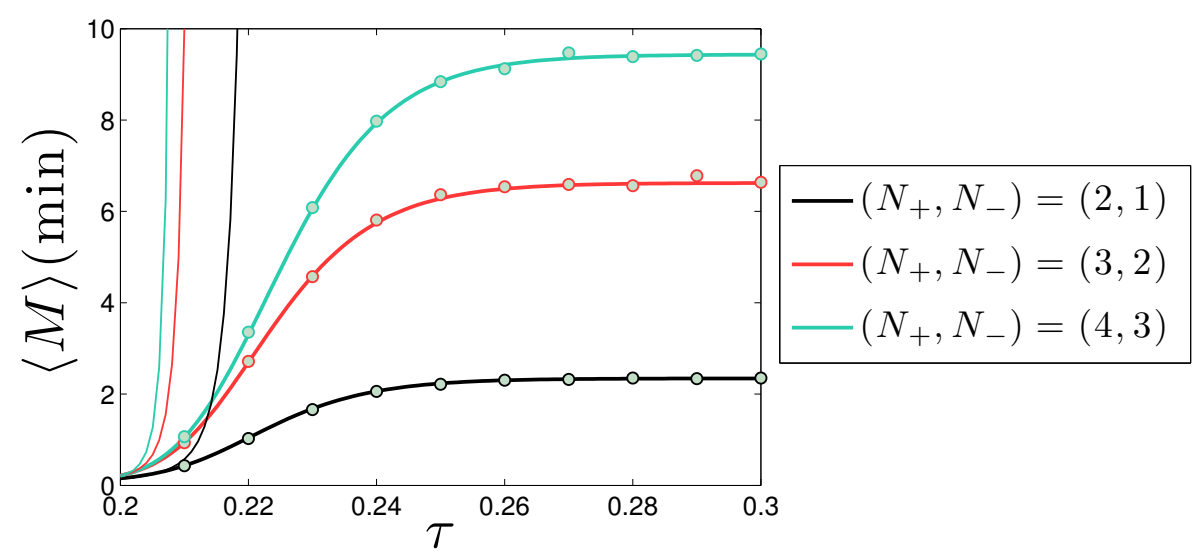

Figure 7. Plot of the mean exit time (MET) for starting position $x_{0}=0$ as a function of $\tau$ for different motor configurations $\left(N_{+}, N_{-}\right)$. Thick solid lines represent analytical solutions to (6.7), thin solid lines represent the exit time $M_{B}$ for a Brownian particle (6.4), and symbols represent averaged Monte-Carlo simulations. Parameters are chosen according to Table 1 with $l=1 \mu \mathrm{m}$ and $k_{0}=0.5 s^{-1}$.

computation time of Monte-Carlo simulation, which are computationally expensive when exit from the effective potential well is a rare event. These results confirm that the average duration of tau-induced random oscillations is on the order of minutes for a range of different motor configurations.

\section{Conclusions and outlook}

In this paper, we have combined a model of microtubule motor-driven transport with an intermittent trapping process to explore how different signaling mechanisms affect cargo localization. The intermittent trapping process we consider involves an immobile trap that must alter the environment in a local region around itself to capture a randomly moving target. The immobile trap represents cellular machinery associated with a synapse in a dendrite that can engage a signaling mechanism to improve its chances of capturing (temporarily sequestering) a motor-driven cargo, such as a mRNA or mitochondria. We explored two such signaling mechanisms that lead to improved activity-dependent cargo localization.

We first explored the possibility of an [ATP] signal created by increased actin dynamics at an active synapse. We found that a local decrease in [ATP] will cause 
a nearby motor-complex to slow down and spend more time receptive to capture. We used our model to calculate the capture probability and MFPT for the case when [ATP] is held fixed away from the target at $10^{3} \mu M$ and decreased in a small region surrounding the target. The results showed that the capture probability can be increased drastically by the [ATP] signal without significantly increasing the MFPT. Although no experimental evidence exists for ATP gradients, steep gradients are theoretically possible especially considering that ATP is heavily buffered and that there are discrete, isolated dendritic compartments associated with a group of synapses.

It was also found that the number of kinesin and dynein motors bound to the cargo affects its response to the $[\mathrm{ATP}]$ signal. The motor configuration $\left(N_{+}, N_{-}\right)$must be balanced to enable the cargo to travel at a high average velocity in background [ATP] levels and at a slow average velocity in the low $[\mathrm{ATP}]$ conditions near the synapse. This was achieved when many dynein and kinesin motors were bound to the cargo, so that the kinesin motors were better able to overpower the weaker dynein motors at high [ATP], while remaining balanced with the dynein motors at low [ATP]. One experimental study showed that vesicles travel with approximately 1-4 kinesin and 1-5 dynein motors bound [53], which is consistent with our results. In another study, many dynein motors worked against a single kinesin motor; however, the dynein motors may have been weaker and more prone to detachement [54].

We also explored a signaling mechanism based on the microtubule-associated protein tau. Using experimental results quantifying the $\tau$-dependent binding rate for kinesin, we calculated the capture probability and MFPT as a function of $\tau$ localized around the synapse. Much like the $[\mathrm{ATP}]$ signal, we found that increasing $\tau$ causes a sharp increase in the capture probability, and we also found that the MFPT was held relatively constant. These results show that, like ATP, tau can also serve as a signaling mechanism to enhance localization of a motor-driven cargo. Interestingly, a recent study [28] has found a link between synaptic activity and MAP2 recruitment to microtubules, mediated by posttranslational microtubule modifications (specifically, by polyglutamylation of tubulin dimers). This study also showed that the upstream signal inducing MAP2-microtubule binding may also affect certain kinesin-cargo linkers; specifically, it showed that gephyrin - a cargo linker for KIF5function was disrupted by polyglutamylation, while the KIF5 cargo linker GRIP1 was unaffected. These observations imply an intricate traffic regulation system capable of localizing specific types of motor-driven cargo.

An unexpected observation of the tau signal's effect on the motor-complex was the response of the drift velocity, derived from the QSS reduction, to high levels of tau. Interestingly, as tau is increased the drift velocity changes sign, creating an effective potential well that confines the motor-driven cargo. The motion of the cargo in this potential well would appear as random saltatory oscillations, which has been observed experimentally in dendritic mRNA transport; recall that dendritic transport would depend on MAP2, which is functionally similar to tau. To explore this, we calculated the average duration of the random oscillations for different motor configurations and various concentrations of tau. Our results showed that the average duration of the oscillations is on the order of minutes, which is consistent with experimental findings. This provides additional support for the "tug-of-war" theory of multiplemotor transport in neurons and further motivates the possibility that tau not only serves as a microtubule stabilizer, but may also provide a means of regulating motor traffic. 
Local synaptic signaling enhances the stochastic transport of motor-driven cargo in neurons 22

\section{Acknowledgments}

This publication was based on work supported in part by the NSF (DMS-0813677) and by Award No KUK-C1-013-4 made by King Abdullah University of Science and Technology (KAUST). PCB was also partially supported by the Royal SocietyWolfson Foundation.

\section{Glossary}

\section{References}

[1] Bean A 2007 Protein trafficking in neurons (Amsterdam: Elsevier/Academic Press) ISBN 9780123694379 (hardback : alk. paper)

[2] Bramham C R and Wells D G 2007 Nat. Rev. Neurosci. 8 776-789

[3] Chang D T W, Honick A S and Reynolds I J 2006 J. Neurosci. 26 7035-7045

[4] Gennerich A and Schild D 2006 Phys. Biol. 3 45-53

[5] Zinsmaier K E, Babic M and Russo G J 2009 Mitochondrial Transport Dynamics in Axons and Dendrites (Results and Problems in Cell Differentiation: 48) (Springer Berlin / Heidelberg)

[6] MacAskill A F and Kittler J T 2010 Trends Cell Biol. 20 102-12

[7] Dynes J L and Steward O 2007 J. Comp. Neurol. $500433-447$

[8] Rook M S, Lu M and Kosik K S 2000 J. Neurosci. 20 6385-6393

[9] Stone M C, Roegiers F and Rolls M M 2008 Mol. Biol. Cell $194122-4129$

[10] Burton P R 1988 Brain Res. 473 107-115

[11] Baas P W, Deitch J S, Black M M and Banker G A 1988 Proc. Natl. Acad. Sci. U.S.A. 85 $8335-8339$

[12] Grafstein B and Forman D S 1980 Physiol. Rev. 60 1167-1283

[13] Knowles R B, Sabry J H, Martone M E, Deerinck T J, Ellisman M H, Bassell G J and Kosik K S 1996 J. Neurosci. 16 7812-7820

[14] Bannai H, Inoue T, Nakayama T, Hattori M and Mikoshiba K 2004 J. Cell Sci. 117 163-175

[15] Welte M 2004 Curr. Biol. 14 R525-R537

[16] Pilling A D, Horiuchi D, Lively C M and Saxton W M 2006 Mol. Biol. Cell 17 2057-2068

[17] Rogers S L, Tint I S, Fanapour P C and Gelfand V I 1997 Proc. Natl. Acad. Sci. U.S.A. 94 $3720-3725$

[18] Müller M J I, Klumpp S and Lipowsky R 2008 Proc. Natl. Acad. Sci. U.S.A. 105 4609-4614

[19] Newby J M and Bressloff P C 2010 Bull. Math. Biol. 27pp

[20] Newby J M and Bressloff P C 2010 J. Stat. Mech. 2010 P04014

[21] Fukazawa Y, Saitoh Y, Ozawa F, Ohta Y, Mizuno K and Inokuchi K 2003 Neuron 38 447-460

[22] Telley I A, Bieling P and Surrey T 2009 Biophys. J. 96 3341-3353

[23] Tokuraku K, Noguchi T Q, Nishie M, Matsushima K and Kotani S 2007 J. Biochem. 141 $585-591$

[24] Kosik K S, Joachim C L and Selkoe D J 1986 Proc. Natl. Acad. Sci. U.S.A. 83 4044-4048

[25] Lebel M, Patenaude C, Allyson J, Massicotte G and Cyr M 2009 Neuropharm. 57 392-402

[26] Shafit-Zagardo B and Kalcheva N 1998 Mol. Neurobiol. 16 149-162

[27] Szebenyi G, Bollati F, Bisbal M, Sheridan S, Faas L, Wray R, Haferkamp S, Nguyen S, Caceres A and Brady S T 2005 Curr. Biol. 15 1820-1826

[28] Maas C, Belgardt D, Lee H K, Heisler F F, Lappe-Siefke C, Magiera M M, van Dijk J, Hausrat T J, Janke C and Kneussel M 2009 Proc. Natl. Acad. Sci. U.S.A. 106 8731-8736

[29] Trinczek B, Ebneth A, Mandelkow E and Mandelkow E 1999 J. Cell Sci. 112 2355-2367

[30] Seitz A, Kojima H, Oiwa K, Mandelkow E M, Song Y H and Mandelkow E 2002 EMBO J 21 4896-4905

[31] Dixit R, Ross J L, Goldman Y E and Holzbaur E L F 2008 Science 319 1086-1089

[32] Vershinin M, Carter B C, Razafsky D S, King S J and Gross S P 2007 Proc. Natl. Acad. Sci. U.S.A. $10487-92$

[33] Müller M J, Klumpp S and Lipowsky R 2008 J. Stat. Phys. 133 1059-1081

[34] Visscher K, Schnitzer M J and Block S M 1999 Nature 400 184-189

[35] Schnitzer M J, Visscher K and Block S M 2000 Nat. Cell Biol. 2 718-723

[36] King S J and Schroer T A 2000 Nat. Cell Biol. 2 20-24

[37] Gao Y Q 2006 Biophys. J. 90 811-821

[38] Mogilner A, Fisher A and Baskin R 2001 J. Theor. Biol. 211 143-157 
Local synaptic signaling enhances the stochastic transport of motor-driven cargo in neurons 23

[39] Kolomeisky A B and Fisher M E 2007 Annu. Rev. Phys. Chem. 58 675-695

[40] Liepelt S and Lipowsky R 2007 Phys. Rev. Lett. 98258102

[41] Carter N J and Cross R A 2005 Nature 435 308-312

[42] Newby J M and Bressloff P C 2009 Phys. Rev. E 80021913

[43] Gardiner C W 1983 Handbook of stochastic methods for physics, chemistry, and the natural sciences vol v. 13 (Berlin: Springer-Verlag) ISBN 0387113576 (U.S.)

[44] Loverdo C, Benichou O, Moreau M and Voituriez R 2009 J. Stat. Mech.-Theory Exp.

[45] Bressloff P C and Newby J 2009 New J. Phys. 11

[46] Othmer H G and Hillen T 2002 SIAM J. Appl. Math. 62 1222-1250

[47] Hillen T 2003 Eur. J. Appl. Math. 14 613-636

[48] Redner S 2001 A guide to first-passage processes (Cambridge, UK: Cambridge University Press) ISBN 0521652480

[49] Chai Y, Lipowsky R and Klumpp S 2009 J. Stat. Phys. 135 241-260 ISSN 0022-4715

[50] Grzeschik H, Harris R J and Santen L 2010 Phys. Rev. E 81031929

[51] Kunwar A, Vershinin M, Xu J and Gross S P 2008 Curr. Biol. 18 1173-1183

[52] Müller M J, Klumpp S and Lipowsky R 2010 Biophys. J. 982610 - 2618

[53] Hendricks A G, Perlson E, Ross J L, Schroeder, H W, Tokito M and Holzbaur E L 2010 Curr. Biol. $20697-702$

[54] Soppina V, Rai A K, Ramaiya A J, Barak P and Mallik R 2009 Proc. Natl. Acad. Sci. U.S.A. 106 19381-19386 



\section{RECENT REPORTS}

15/10 Travelling waves in hyperbolic chemotaxis equations Xue

Hwang

Painter

Erban

16/10 The Physics and Mechanics of Biological Systems

Goriely

Moulton

17/10 Crust formation in drying colloidal suspensions

Style

Peppin

18/10 A Mathematical Model of Tumor-Immune Interactions

Robertson-Tessi

El-Kareh

Goriely

19/10 Elastic cavitation, tube hollowing, and differential growth in plants and biological tissues

Goriely

Moulton

Vandiver

20/10 Asymptotic expressions for the nearest and furthest dislocations in a pile-up against a grain boundary

21/10 Cardiac electromechanics: the effect of contraction model on the mathematical problem and accuracy of the numerical scheme

Hall

Pathmanathan

Chapman

Gavaghan

Whiteley

22/10 Fat vs. thin threading approach on GPUs: application to stochastic simulation of chemical reactions

Klingbeil

Erban

Giles

Maini

23/10 Asymptotic analysis of a system of algebraic equations arising in dislocation theory

Hall

Chapman

Ockendon

25/10 Preconditioning for Allen-Cahn Variational Inequalities with Non-

Blank Local Constraints

Sarbu

Stoll

26/10 On an evolution equation for sand dunes Ellis

Fowler

27/10 On Liquid Films on an Inclined Plate

Benilov

Chapman

McLoed

Ockendon

Zubkov

28/10 An a posteriori error analysis of a mixed finite element Galerkin

Memon approximation to second order linear parabolic problems

Nataraj

Pani

29/10 A Priori Error Estimates for Semidiscrete Finite Element ApproxGoswami imations to Equations of Motion Arising in Oldroyd Fluids of Or-

Pani der One

30/10 The Landau-de Gennes theory of nematic liquid crystals: Uniaxiality versus Biaxiality 
32/10 Nonlinear instability in flagellar dynamics: a novel modulation

Gadelha mechanism in sperm migration?

Gaffney

Smith

Kirkman-Brown

33/10 Error bounds on block GaussSeidel solutions of coupled multiphysics problem

Whiteley

Gillow

Tavener

Walter

34/10 A random projection method for sharp phase boundaries in lattice

Reis Boltzmann simulations

Dellar

35/10 Regularized Particle Filter with Langevin Resampling Step

Duan

Farmer

Moroz

36/10 Sequential Inverse Problems Bayesian Principles and the Logistic

Duan Map Example

Farmer

Moroz

37/10 Circumferential buckling instability of a growing cylindrical tube Moulton

Goriely

38/10 Preconditioners for state constrained optimal control problems Stoll with Moreau-Yosida penalty function

Wathen

Copies of these, and any other OCCAM reports can be obtained from:

Oxford Centre for Collaborative Applied Mathematics Mathematical Institute

24 - 29 St Giles'

Oxford

OX1 3LB

England

www.maths.ox.ac.uk/occam 